\title{
Unidad didáctica para fortalecer la competencia indagación en biología de los estudiantes del grado noveno en la institución educativa Juan Pablo I
}

\author{
Didactic unit to strengthen the competence in biology \\ research of ninth grade students at the Juan Pablo I \\ educational institution
}

\begin{abstract}
Unidade didática para fortalecer a competência em pesquisa em biologia de alunos do nono ano do centro universitário Juan Pablo I
\end{abstract}

\author{
Ludy Adriana Bateca Arias \\ Candidata a magister en educación \\ Universidad Autónoma de Bucaramanga \\ lbateca@unab.edu.co \\ Elvira Torrado Santamaría \\ Magister en química \\ Universidad Autónoma de Bucaramanga \\ etirado@unab.edu.co
}

\section{Resumen}

Para fortalecer la competencia indagación en biología, se tuvo en cuenta un enfoque cualitativo, a través de la investigación acción. El proceso de investigación se desarrolló en tres fases: prueba escrita, diseño e implementación de actividades y reflexión; mediante la prueba escrita se estableció el nivel de la competencia de indagación en biología, en los estudiantes del grado noveno, posteriormente se diseñó la unidad didáctica para fortalecer dicha competencia, luego se implementaron las actividades propuestas en la unidad didáctica, por último se reflexiona sobre la efectividad de la estrategia pedagógica implementada. Durante la observación en las intervenciones, se reflexionó sobre los cambios del estudiante en el transcurso de las actividades, donde se pudo obtener mayor disposición y participación en las clases. 
Palabras claves: unidad didáctica, fortalecer, aprendizaje, competencia, indagación.

\begin{abstract}
To strengthen the competence of research in biology, a qualitative approach was taken into account, through action research. The research process was developed in three phases: written test, design and implementation of activities and reflection; by means of the written test the level of the competence of investigation in biology was established, in the students of the ninth grade, later the didactic unit was designed to strengthen said competence, then the activities proposed in the didactic unit were implemented, finally it is reflected on the effectiveness of the pedagogical strategy implemented. During the observation in the interventions, we reflected on the changes of the student in the course of the activities, where greater disposition and participation in the classes could be obtained.
\end{abstract}

Keywords: didactic unit, strengthen, learning, competence, inquiry.

\title{
Resumo
}

Para reforçar a competência de pesquisa em biologia, uma abordagem qualitativa foi levada em conta, através da pesquisa-ação. O processo de pesquisa foi desenvolvido em três fases: teste escrito, desenho e implementação de atividades e reflexão; por meio do teste escrito foi estabelecido o nível da competência de investigação em biologia, nos alunos do nono ano, posteriormente a unidade didática foi projetada para reforçar tal competência, então as atividades propostas na unidade didática foram implementadas, enfim se reflete em a eficácia da estratégia pedagógica implementada. Durante a observação nas intervenções, refletimos sobre as mudanças do aluno no decorrer das atividades, onde maior disposição e participação nas aulas puderam ser obtidas.

Palavras-chave: unidade didática, fortalecer, aprender, competência, questionamento.

\section{Introducción}

Para los procesos pedagógicos es importante tener en cuenta cómo actúan los actores del proceso: los estudiantes en el salón de clase, el docente en su práctica pedagógica y la colaboración de los padres de familia en el desarrollo de aprendizaje de sus hijos, por lo que es importante identificar las fortalezas, debilidades y ventajas se cuentan en el aprendizaje de los estudiantes. 
El objetivo general es fortalecer la competencia indagación en biología en los estudiantes del grado noveno mediante la estrategia unidad didáctica.

El desarrollo de la investigación se orientó bajo el enfoque cualitativo con fundamento constructivista, desde el aprendizaje significativo. Desde la investigación acción se planteó en fases, las cuales fueron orientadas desde los aportes teóricos y procesos investigativos en tres fases: fase prueba escrita, fase de diseño e implementación y fase de reflexión de las actividades.

Se dan las conclusiones de la propuesta de investigación, con referencia a cada una de las intervenciones realizadas durante propuesta a través de la unidad didáctica y las actividades innovadoras y creativas con el fin de fortalecer la competencia indagación en los estudiantes del grado noveno de la institución educativa Juan Pablo I.

\section{Marco conceptual}

Para el diseño y análisis del desempeño de los estudiantes, después de las intervenciones usando la unidad didáctica como estrategia de enseñanza, se tuvieron en cuenta los lineamientos del ICFES en el área de ciencias naturales (2007), concepto de la competencia indagar de Wells (2001) y del ICFES, competencias en ciencias naturales.
Las ciencias naturales son una disciplina, la cual presenta especificidades, que les permiten manejar lenguajes técnicos y procesos específicos en su enseñanza, por lo tanto se definen en ciencias Naturales siete competencias básicas (Documento ICFES, lineamientos del área de Ciencias Naturales, 2007) que son:

1. Identificar: capacidad para reconocer y diferenciar fenómenos, representaciones y preguntas pertinentes sobre estos fenómenos.

2. Indagar: capacidad para plantear preguntas $y$ procedimientos adecuados $\mathrm{y}$ para buscar, seleccionar, organizar e interpretar información relevante para dar respuesta a esas preguntas.

3. Explicar: capacidad para construir y comprender argumentos, representaciones 0 modelos que den razón de fenómenos.

4. Comunicar: capacidad para escuchar, plantear puntos de vista y compartir conocimiento.

5. Trabajar en equipo: capacidad para productivamente asumiendo compromisos.

6. Disposición para reconocer la dimensión social del conocimiento.

7. Disposición para aceptar la naturaleza cambiante del conocimiento.

\section{Competencia indagar}

La indagación es una aproximación a los temas y 
problemáticas escogidas en los cuales se promueve formular preguntas reales, cuando éstos ocurren y por quien sea que pregunte. Igualmente importante, como garantía de calidad de una aproximación a la indagación todas las respuestas tentativas se toman seriamente y se investigan tan rigurosamente como las circunstancias lo permitan (Gordon Wells, 2001, Indagación dialógica: hacia una teoría y una práctica socioculturales de la educación).

La competencia científica indagar se propone para lograr una alfabetización científica y se define como. "La capacidad para formular preguntas y procedimientos adecuados con el fin de buscar, seleccionar, organizar e interpretar información relevante y así dar respuesta a esas preguntas" (ICFES, grupo de evaluación de la educación básica y media, 2016). Así mismo Garnica y Arteta (2010) exponen que el trabajo de las competencias científicas como indagar y explicar son:

"Las competencias explicar e indagar dan cuenta de una forma particular del conocimiento (...) por ser una forma de realización específica de la comprensión de los fenómenos y del quehacer en el área, el desarrollo de estas competencias permite que el estudiante vaya avanzando paulatinamente en el conocimiento del mundo desde una óptica que depende de la posibilidad de dudar, de preguntarse acerca de lo que se observa para interactuar de manera lógica y propositiva en el mundo en que se desarrolla" (Sonia Garnica Mojica y Judith Arteta De Molina, 2010); el proceso de indagación en el aula de clase, nos permite involucrar procesos como observación de problemas reales o del contexto, elaboración de hipótesis, plantear múltiples actividades o diseñar estrategias, generar datos e información, analizarlos, evaluar y comunicar, lo que permite que el aprendizaje sea significativo, ya que se va construyendo por etapas y además desarrolla habilidades comunicativas y trabajo en equipo.

La educación colombiana en todos sus niveles (Prueba Saber) propone evaluar tres competencias específicas: Uso comprensivo del conocimiento científico, explicación de fenómenos e indagación; ya que busca dar cuenta de la capacidad de los estudiantes para utilizar sus conocimientos básicos en ciencias naturales para la comprensión y resolución de problemas, esto se realizó en la alineación de las pruebas11. El Instituto Colombiano para la Evaluación de la Educación Superior ICFES, es una entidad adscrita al Ministerio de Educación Nacional, es la encargada de promover y evaluar, que define la indagación como: "Es la capacidad para formular preguntas y procedimientos adecuados con el fin de buscar, seleccionar, organizar e interpretar información relevante y así dar respuesta a esas preguntas" (ICFES, grupo de evaluación de la educación básica y media, 2016). 
La educación en ciencias busca promover una forma de trabajo propia de las ciencias como un tipo particular de indagación en el que se parte de una pregunta pertinente $y$ se establecen los elementos que deben ser considerados para resolverla (lo cual implica apoyarse en la información fáctica, en el conocimiento adquirido y en la capacidad de crear o imaginar estrategias de solución posibles). Una vez se ha logrado formular una pregunta relativamente precisa, se puede proceder a establecer un método de trabajo para resolverla. Incluye, además, la acción planeada, orientada a la búsqueda de información que ayude a establecer la validez de una respuesta preliminar y la planeación de un experimento sencillo (ICFES, 2006).

\section{Unidad Didáctica}

La unidad didáctica es una forma de planificar el proceso de enseñanza-aprendizaje alrededor de un elemento de contenido que se convierte en eje integrador del proceso, aportándole consistencia y significatividad. Esta forma de organizar conocimientos y experiencias debe considerar la diversidad de elementos que contextualizan el proceso (nivel de desarrollo del estudiante, medio sociocultural y familiar, Proyecto Curricular, recursos disponibles) para regular la práctica de los contenidos, seleccionar los objetivos básicos que pretende conseguir, las pautas metodológicas con las que trabajará, las experiencias de enseñanza-aprendizaje necesarios para perfeccionar dicho proceso" (Escamilla, 1995).

\section{Elementos de una unidad didáctica}

Para el desarrollo de la unidad didáctica se requieren ciertos elementos que permitan su elaboración por la que se emplearan los siguientes: (Tomado del módulo I. Explorando preguntando - Ciencia en la escuela 2012).

Descripción: indicar el tema o el nombre de la unidad didáctica, así como los conocimientos previos que deben tener los estudiantes, las actividades de motivación, etc. También indicar el número de sesiones y el momento en el que se pondrá en práctica.

Objetivos: establecer los objetivos didácticos para ver los lo que los estudiantes van a adquirir con la unidad. Se enfocan en el ¿qué? Y el ¿para qué?

Contenidos: hablar sobre los contenidos de aprendizaje, relativos a conceptos, procedimientos y actitudes.

Secuencia de actividades: establecer la secuencia de aprendizaje, de forma que las actividades estén relacionadas entre sí.

Recursos o materiales: indicar los diferentes recursos específicos 
que son necesarios para desarrollar la unidad didáctica paso a paso.

Organización de espacio y tiempo: se recomienda indicar los aspectos concretos relacionados con la organización del espacio y el tiempo que requiere la unidad didáctica.

Evaluación: indicar cuáles serán los criterios e indicadores de la valoración de los aprendizajes de los estudiantes.

\section{Constructivismo}

Piaget considera que "el
desarrollo cognoscitivo es un
proceso continuo y biológico,
siendo un proceso de
reconstrucción constante, en
donde el pensamiento va
evolucionando y adaptándose a su
realidad. Esta adaptación de
aprendizaje requiere de dos
procesos complementarios: asimilación y acomodación". En su teoría Piaget tiene como principal objetivo explicar no sólo cómo conocemos el mundo sino también cómo cambia nuestro conocimiento sobre ese mundo. La asimilación se refiere al modo en que un organismo se enfrenta a un estímulo del entorno en términos de organización actual, mientras que la acomodación implica una modificación de la organización actual en respuesta a las demandas del medio. Mediante la asimilación y la acomodación vamos reestructurando cognitivamente nuestro aprendizaje a lo largo del desarrollo de las temáticas.

Para la educación se debe tener como meta principal "Crear personas que sean capaces de innovar, no simplemente de repetir lo que otras generaciones han hecho. Personas que sean creativas, inventoras $y$ descubridoras. La segunda meta de la educación es la de formar mentes que sean críticas, que puedan verificar y no aceptar todo lo que se les trasmite como válido o verdadero" (Piaget, 1985).

\section{Aprendizaje significativo}

Es el proceso según el cual se relaciona un nuevo conocimiento o información con la estructura cognitiva del que aprende de forma no arbitraria y sustantiva o no literal. Esa interacción con la estructura cognitiva no se produce como un todo, sino con aspectos relevantes presentes en la misma, que reciben el nombre de ideas de anclaje (Ausubel, 1976).

El estudiante debe manifestar
$[\ldots]$ una disposición para
relacionar sustancial y no arbitrariamente el nuevo material con su estructura cognoscitiva, como que el material que aprende es potencialmente significativo para él, es decir, relacionable con su estructura de conocimiento sobre una base no arbitraria (Ausubel, 1983), durante este proceso el estudiante va reconstruyendo y reajustando para posibilitar un nuevo aprendizaje. 
Ausubel distingue tres tipos de aprendizaje significativo:

Aprendizaje de representaciones: consiste en la atribución de significados a determinados símbolos. Ocurre cuando se igualan en significado símbolos arbitrarios con sus referentes (objetos, eventos, conceptos) y significan para el estudiante cualquier significado al que sus referentes aludan (Ausubel, 1983). El aprendizaje de representaciones consiste en retener el nombre de las palabras y otros símbolos, y asociarlos con lo que representan. Cuando uno aprende una palabra, ésta es carente de todo sentido, sin saber lo que representa, ya que podría haberse llamado de otro modo. Aprender representaciones es asignar un símbolo a una idea. Ver una pelota y saber que se llama pelota.

Aprendizaje de conceptos: los conceptos se definen como "objetos, eventos, situaciones o propiedades de que posee atributos de criterios comunes y que se designan mediante algún símbolo o signos" (Ausubel, 1983) de acuerdo con ello podría decirse que también es un aprendizaje de representaciones.

Los conceptos son adquiridos a través de dos procesos: formación y asimilación.

En la formación de conceptos, las características del concepto se adquieren a través de la experiencia directa, en sucesivas etapas de formulación y prueba de hipótesis.

Por aprendizaje por asimilación entendemos el proceso mediante el cual " la nueva información es vinculada con aspectos relevantes y pre-existentes en la estructura cognoscitiva, proceso en que se modifica la información recientemente adquirida y la estructura pre-existente (Ausubel, 1983).

En este punto es donde cobra valor la práctica del laboratorio, ya que es en este momento donde el estudiante puede aplicar lo explicado por el profesor durante una clase, demostrar la veracidad o falsedad de una hipótesis, comprobar un resultado, comparar y llevar la teoría a la práctica para afianzar sus conocimientos previos con los nuevos.

Aprendizaje de proposiciones: este tipo de aprendizaje va más allá de la simple asimilación de lo que representan las palabras, combinadas o aisladas, puesto que exige captar el significado de las ideas expresadas en forma de proposiciones.

El aprendizaje de proposiciones implica la combinación y relación de varias palabras cada una de las cuales constituye un referente unitario, luego estas se combinan de tal forma que la idea resultante es más que la simple suma de los significados de las palabras componentes individuales, produciendo un nuevo significado 
que es asimilado a la estructura cognoscitiva.

\section{Metodología}

La investigación que se realiza lleva un interés pedagógico puesto que su objetivo es fortalecer la competencia indagación en biología con los estudiantes del grado noveno.

\section{Diseño de la investigación}

El proyecto se desarrolló en el marco de una investigación cualitativa mediante la investigación acción, basado en el autor Kemmis (1984), quien ve la investigación acción como "una forma de indagación autoreflexiva realizado por quienes participan (profesorado, alumnado, o dirección) en las situaciones sociales (incluyendo las educativas) para mejorar la racionalidad y la justicia de: a) sus propias prácticas sociales $\mathrm{o}$ educativas; b) su comprensión sobre las mismos; y c) las situaciones e instituciones en que estas prácticas se realizan (aulas o escuelas, por ejemplo) (Torrecilla, 2011).

Kemmis y McTaggart (1988) han descrito las características de la investigación-acción, de la siguiente manera:

- Es participativa, las personas trabajan con la intención de mejorar sus propias prácticas.

- La investigación sigue una espiral introspectiva: una espiral de ciclos de planificación, acción, observación y reflexión.

- Es colaborativa, se realiza en grupo por las personas implicadas.

- Crea comunidades autocríticas de personas que participan y colaboran en todas las fases del proceso de investigación.

- Es un proceso sistemático de aprendizaje, orientado a la praxis (acción críticamente informada y comprometida).

- Induce a teorizar sobre la práctica.

- Somete a prueba las prácticas, las ideas y las suposiciones.

- Implica registrar, recopilar, analizar nuestros propios juicios, reacciones e impresiones en torno a lo que ocurre; exige llevar un diario personal en el que se registran nuestras reflexiones.

- Es un proceso político porque implica cambios que afectan a las personas.

- Realiza análisis críticos de las situaciones.

- Procede progresivamente a cambios más amplios.

- Empieza con pequeños ciclos de planificación, acción, observación y reflexión, avanzando hacia problemas de más envergadura; la inician pequeños grupos de colaboradores, expandiéndose gradualmente a un número mayor de personas. 


\section{Metodología indagatoria}

La idea central de la metodología indagatoria es propiciar una estrategia de enseñanza y aprendizaje que parta de la observación de la realidad, interacción con problemas concretos, propiciándose preguntas referentes a esa realidad que promuevan la búsqueda de información y la experimentación, por ende la construcción activa de su aprendizaje. La aplicación de esta metodología requiere de un proceso sistemático, por lo que a lo largo de su aplicación en diferentes países, se ha requerido de componentes y etapas especificas su implementación, a continuación se describirán de manera general las etapas que se han desarrollado. (Uzcátegui \& Betancourt, 2013).

\section{Etapas para la aplicación de la metodología indagatoria}

La etapa de focalización, es la primera etapa, por ende la crucial para el desarrollo de la metodología, en ella se debe propiciar el interés y la motivación en el estudiante sobre una situación problema. Está basada en la contextualización de una situación, esto se puede dar mediante la observación, el relato de un evento de la comunidad o la presentación de una situación desconocida, seguida de una pregunta bien diseñada que promueva el interés de los estudiantes y la necesidad de resolverla. Su desarrollo debe ser individual, a modo de extraer las concepciones y conocimientos previos que posee el estudiante sobre el tema central del problema y hacer los ajustes pertinentes a su panificación para lograr una construcción efectiva del aprendizaje.

La etapa de exploración, es la que va a propiciar el aprendizaje, en ella los estudiantes desarrollan su investigación, se fundamentan en sus ideas y buscan estrategias para desarrollar experiencias que los lleven a conseguir resultados. Es importante que los estudiantes elaboren sus procedimientos y el docente sirva sólo de guía, permitiendo la argumentación, razonamiento y confrontación de sus puntos de vista.

La etapa de comparación o reflexión, es donde se requiere la participación activa del estudiante. El deberá confrontar la realidad de los resultados observados con sus predicciones, formulando sus propias conclusiones. El docente por su parte, debe estar atento para introducir términos y conceptos que considere adecuados, mediar para que reflexione $y$ analice detalladamente sus conclusiones. Las conclusiones deben presentarse de forma oral y escrita con un lenguaje sencillo, donde el estudiante incluya los conceptos y términos que crea necesarios.

La etapa de aplicación, es la confirmación del aprendizaje, en ella el estudiante debe ser capaz de extrapolar el aprendizaje a eventos cotidianos, generando pequeñas investigaciones o extensiones del trabajo experimental. 
La etapa de evaluación, se encuentra implícita en todas las anteriores, y debe estar centrada en las competencias y destrezas que los estudiantes logran. La evaluación tiene un carácter formativo parcial, que permite monitorear el aprendizaje del estudiante, llevar un seguimiento de la transformación del conocimiento, desde la etapa de focalización hasta la de aplicación. Esta se desarrolla mediante apuntes, observaciones o con ayuda del cuaderno de trabajo, contrastando los resultados obtenidos con una escala que gradúa las habilidades básicas que deben lograr los estudiantes.

Las evaluaciones sumativas surgen principalmente de narraciones orales o escritas que demuestren lo aprendido, su relación con otros conocimientos y formas de analizar las ideas, siendo el instrumento ideal para recolectar información las rubricas que especifiquen las habilidades cognitivas que se desean evaluar en los estudiantes, estas observaciones son tomadas preferiblemente en los escritos que deja el estudiante en sus cuadernos de trabajo o en la entrega de informes.

\section{Fases de la investigación}

El proceso del proyecto de investigación inició como:

Primera fase la elaboración y aplicación de prueba escrita tipo prueba saber, diseñado con diez preguntas, extraído de las Pruebas
Saber de años anteriores, para identificar las falencias en la competencia de indagación en biología, con los estudiantes del grado noveno 01 , de la institución educativa Juan Pablo I.

Segunda fase de la investigación se realiza la aplicación de la estrategia pedagógica, teniendo como pauta la observación para determinar el comportamiento, las actitudes, aceptación y logros de los estudiantes ante la intervención con las actividades.

Tercera fase se basó en la aplicación de la estrategia y del desarrollo del trabajo en cada uno de los tiempos pedagógicos, exaltando el buen desempeño e interés de los estudiantes en las actividades propuestas.

Por último y como fase cuarta, se realiza el análisis de los resultados de acuerdo a los objetivos propuestos para el fortalecimiento de la competencia indagación en biología del grado noveno.

\section{Técnicas e instrumentos para la recolección de la información}

Para realizar la ejecución del proyecto de investigación se emplearon las siguientes técnicas e instrumentos que tienen como fin el cumplimiento de los objetivos planteados con miras al rendimiento académico de la institución. Entre los instrumentos que se aplicaron se encuentran los siguientes: técnicas de observación, prueba escrita, la 
entrevista, la encuesta y el diario pedagógico.

\section{Prueba escrita}

Como diagnóstico se realizó una prueba tipo test de diez preguntas, que fueron extraídas de las Pruebas Saber de los años anteriores, preguntas correspondientes a la competencia indagación de biología del grado noveno, la prueba fue evaluada de 1 a 10 , donde se estableció cuatro niveles: 1-3 bajo, 4-5 mínimos, 6-7 satisfactorio y 8-9 avanzado y se logró identificar el número de aciertos. La prueba arrojo un bajo nivel de los estudiantes en la competencia indagación en biología.

\section{La observación}

La observación es uno de los métodos y técnicas de recogida de datos más utilizados en la investigación educativa. Bassedas, Coll y otros (1984:20) manifiestan: "la observación perspicaz, rigurosa y sistemática es el instrumento indispensable para comprender el comportamiento del alumno en el trascurso de las tareas de aprendizaje y para modificar su contenido y presentación en consecuencia. La observación adquiere casi papel preponderante y los problemas de qué observar y cómo observar se convierten en las cuestiones esenciales de la evaluación formativa en el parvulario y en el ciclo inicial". (PUEBLA, 2010).
En la investigación se utilizó la observación como instrumento para recolectar cada uno de los comportamientos desenvolvimiento en las actividades pedagógicas por parte de los estudiantes, se pudo reflexionar, en alguna de las actividades en donde, no todos los estudiantes, afrontan el proceso educativo de la misma forma.

\section{Diario pedagógico}

Este instrumento es primordial para la investigación, ya que aquí se consignan cada una de las actitudes observadas en los estudiantes, al momento de realizar las actividades propuestas, consta de un folder en el cual se escriben todos los apuntes relevantes de la práctica pedagógica, en donde también se puede hacer una reflexión para mejorar esta práctica o cualquier situación presentada. Este diario se llevará durante toda la práctica de la investigación y es de primordial importancia para el análisis de la información.

\section{Resultados y discusión}

Al inicio de la investigación se realizó una prueba escrita tipo prueba SABER, para determinar el nivel de la competencia indagación en los estudiantes del grado noveno, se estableció cuatro niveles: 1-3 bajo, 4-5 mínimos, 6-7 satisfactorio y 8-9 avanzado.

Se encontró que de los 32 estudiantes a los que se les aplicó la prueba escrita, 13 se valoraron 
con un nivel bajo, 13 en un nivel mínimo, 5 en un nivel satisfactorio y 1 un nivel avanzado.

Las actividades desarrolladas sobre Genética Mendeliana permitieron fortalecer en los estudiantes la competencia indagación, ya que el planteamiento de las clases favoreció en la formulación de preguntas, la discusión de interrogantes y la solución de situaciones cotidianas. Al iniciar la primera actividad se sentía el desapego y falta de interés de los estudiantes; no sabían que era "la competencia indagación" y como era que estaban fallando en ella. No fue sencillo hacer que los estudiantes tuvieran buenas disposición por la actividad, ya que cuestionarse de como ellos interpretan, no es algo que les agrade, finalmente al terminar las dos primera intervenciones se evidencio el cambio de actitud de los estudiantes sin ninguna clase de presión o apreciativa de nota.

En las actividades aplicadas también se evidenció el buen humor de los estudiantes al momento de realizar coplas, trabalenguas y adivinanzas, aunque cuando se les pide que hagan producciones propias, presentan un poco de dificultad en realizar ejercicios con instrucciones ya que no interpretan bien la guía.

$\mathrm{Al}$ inicio de las intervenciones los estudiantes no se enfocaban en la pregunta que se realizaba y tampoco utilizaban un patrón o secuencia para dar respuesta a aquello que se le indicaba, poco a poco fueron adquiriendo la habilidad de ir más allá de lo que podían ver, al terminar las actividades la mayoría de los estudiantes cuestionaban, preguntaban, participaban, proponían e indagaban.

No todos los estudiantes hicieron este progreso, ya que algunos faltan frecuentemente a clase, no prestan atención clase por estar hablando con los demás compañeros, aunque se trató de cambiar el comportamiento de estos estudiantes con dos no se logró avanzar mucho.

La estrategia pedagógica escogida fue asertiva ya que fue una forma muy ordenada y sencilla de aplicar, cada actividad estuvo descrita y llevada a cabo con los lineamientos que se fundamentó, bajo el constructivismo y el aprendizaje significativo, también en cada una de las actividades se aplicó el modelo indagatorio.

Los contenidos en las actividades tuvieron acogida $y$ entendimiento por los estudiantes, ya que no eran actividades que fueran de mayor dificultad, se trató de utilizar actividades que manejaran y fueran un aprendizaje cotidiano, dando paso, así, a construir un verdadero aprendizaje significativo. Se realizó el modelo indagatorio ya que las clases no pueden ser una rutina $y$ mucho menos algo predecible, ya que esto aburre al estudiante. 
En un inicio se implementó las actividades para trabajar de forma individual, aunque algunas de ellas debían hacer en cooperación, pero los estudiantes propusieron hacerse en grupos, lo que fue una buena propuesta ya que constantemente discutían y se cuestionaba el aprendizaje de cada uno.

Las actividades fueron revisadas grupo por grupo y así se resolvían algunos interrogantes que tenían los estudiantes, se hacía corrección de las actividades que lo requerían y de las que no se hacía conversatorio en el salón de clase para saber la opinión de cada grupo.

Es satisfactorio cuando un estudiante trabaja sin el afán de una nota, simplemente lo realiza por querer obtener conocimiento y resolver sus propios interrogantes. Las actividades fueron programadas para un tiempo establecido el cual se cumplió a cabalidad.

El docente debe salirse de su clase magistral y ser más creativo e innovador, pero siempre debe tener en cuenta, que sus gustos, no son los mismos que el de los estudiantes, por lo que es importante como docentes analizar cómo estamos realizando el proceso de enseñanza, ya que si se utiliza la innovación como herramienta para la enseñanza el estudiante se motiva por el aprender, de lo contrario estaríamos perdiendo estudiantes del aula de clase, ya que cada uno de ellos tienen infinitos de problemas y situaciones que resolver.

La evaluación del estudiante debe ser constante y basado en lo cotidiano en su contexto ya que el estudiante con cada actividad va demostrando sus avances en el conocimiento.

\section{Conclusiones}

En respuesta a la pregunta problema formulado en la investigación, se puede concluir que, durante la observación que se realizó en cada una de las intervenciones pedagógicas, se logró fortalecer la competencia indagación en biología del grado noveno a través de la implementación de la Unidad didáctica. Ya que el desarrollo de las actividades pedagógicas como: lecturas, adivinanzas, coplas, videos, chistes, trabalenguas, gráficos, carteleras, mapas mentales, trabajo en grupo, etc. Llevo al estudiante que cuestionara, preguntara, participara, propusiera e indagara, con respecto al tema que se iba trabajando.

De acuerdo al primer objetivo propuesto en la investigación se identificó el nivel de desarrollo en el que se encontraban los estudiantes ante la competencia indagación, por lo que se concluyó que los estudiantes efectivamente si presentaban dificultades en la competencia indagación en biología. Puesto que 26 de los 32 estudiantes de la muestra tenían 
dificultad en formular preguntas y procedimientos, en seleccionar, organizar e interpretar información relevante y así dar respuesta a interrogantes.

Respondiendo al segundo objetivo planteado, se elaboró la Unidad Didáctica y en la implementación se planeó cada una de las intervenciones, siendo esta la puerta para que el estudiante fortaleciera la competencia de indagación, también se presentó un ambiente tranquilo, relajado y de confianza, logrando que los estudiantes demostraran el ingenio, la creatividad y el dinamismo por formular preguntas, procedimientos e información relevante acerca de la biología, los estudiantes lograron familiarizarse con el lenguaje técnico respecto a la Genética Mendeliana junto con toda la conceptualización sobre su composición.

Con base en las observaciones realizadas en cada intervención, se puede concluir la importancia del desarrollo de actividades que fortalezcan la competencia indagación y no es solo para el área de biología; también en otros ámbitos les ayuda a entender muchos aspectos del mundo actual, los estudiantes y las personas en general no se plantean cuestionamientos de las situaciones que se presentan a diario por que no vemos más allá de lo simple y esto hace que creamos en lo primero que ven nuestros ojos; y se desconozca su complejidad.

Se logró despertar en los estudiantes el interés por el conocimiento, por el saber, por dar respuesta a sus interrogantes y los planteados por el docente, esto se concluye a partir de las observaciones, ya que el estudiante de inicio a las de las actividades fue muy distinto al de terminar las actividades. Es muy importante la motivación de los estudiantes y así se obtendrán mejores resultados en las pruebas de estado, esto beneficia a los estudiantes y a toda la comunidad educativa. Por lo que se puede decir que se cumplieron con las metas propuestas en la investigación, siendo un proceso continuo para mejoramiento de todos los procesos de enseñanzaaprendizaje involucrados en el acto educativo.

La guía de actividades fue una herramienta importante en la planeación de las diferentes actividades, porque sirvió como directriz a los estudiantes, mostrándoles la información que necesitaban adquirir para dar respuesta a los cuestionamientos formulados; además que fueron presentados de una forma diferente de ver las temáticas propias del área de biología.

Uno de los aspectos más importante que se pudo evidenciar en la práctica de las actividades, es que el docente, debe ser más innovador y creativo en las clases, mostrando escenarios pedagógicos que le permitan al estudiante 
dispuesto en el aula de clases a realizar las actividades que los llevan al desarrollo de sus competencias de indagación en el área de biología.

\section{Referencias}

Ausubel, D. (1976). La educación y la estructura del conocimiento. Investigaciones sobre el proceso de aprendizaje y la. Buenos Aires: Ed. El Ateneo.

Ausubel, D. (1983). Teoría del aprendizaje significativo. México: Trillas.

Escamilla, A. (1995). Unidades Didácticas Una Propuesta De Trabajo En El Aula. Madrid: Luis Vives (EDELVIVES).

Garnica Mojíca, S. E., \& Arteta de Molina, J. (2010). Evaluación del desarrollo de las competencias científicas explicar e indagar en la aplicación de un trabajo práctico sobre fotosíntesis. Biografía: Escritos sobre la Biología y su Enseñanza. Bogotá.

ICFES. (2006). Subdirección Académica Grupo De Evaluación De La Educación Básica Y Media Área En Ciencias Naturales. Bogotá.

ICFES. (2016). Grupo de evaluación de la educación básica y media.

Educativa José María Vélaz, Medellín. Medellín. Obtenido de http://bdigital.unal.edu.co/47 002/1/43207801.2015.pdf
Piaget, J. (1985). La construcción de lo real en el niño.

Puebla, S. B. (2010). Métodos De Investigación En Educación.

Torrecilla, F. J. (2011). Métodos de investigación en Educación Especial.

Uzcátegui, Y., \& Betancourt, C. (2013). La metodología indagatoria en la enseñanza de las ciencias: una revisión de su creciente implementación a nivel de educación básica y media. Caracas. Obtenido de http://revistas.upel.edu.ve/ind ex.php/revinvest/article/viewF ile/3208/1519

Wells, G. (2001). Indagación Dialógica: Hacia Una Teoría Y Una Practica Sociocultural De La Educación. Barcelona: Paidos Ibérica. 


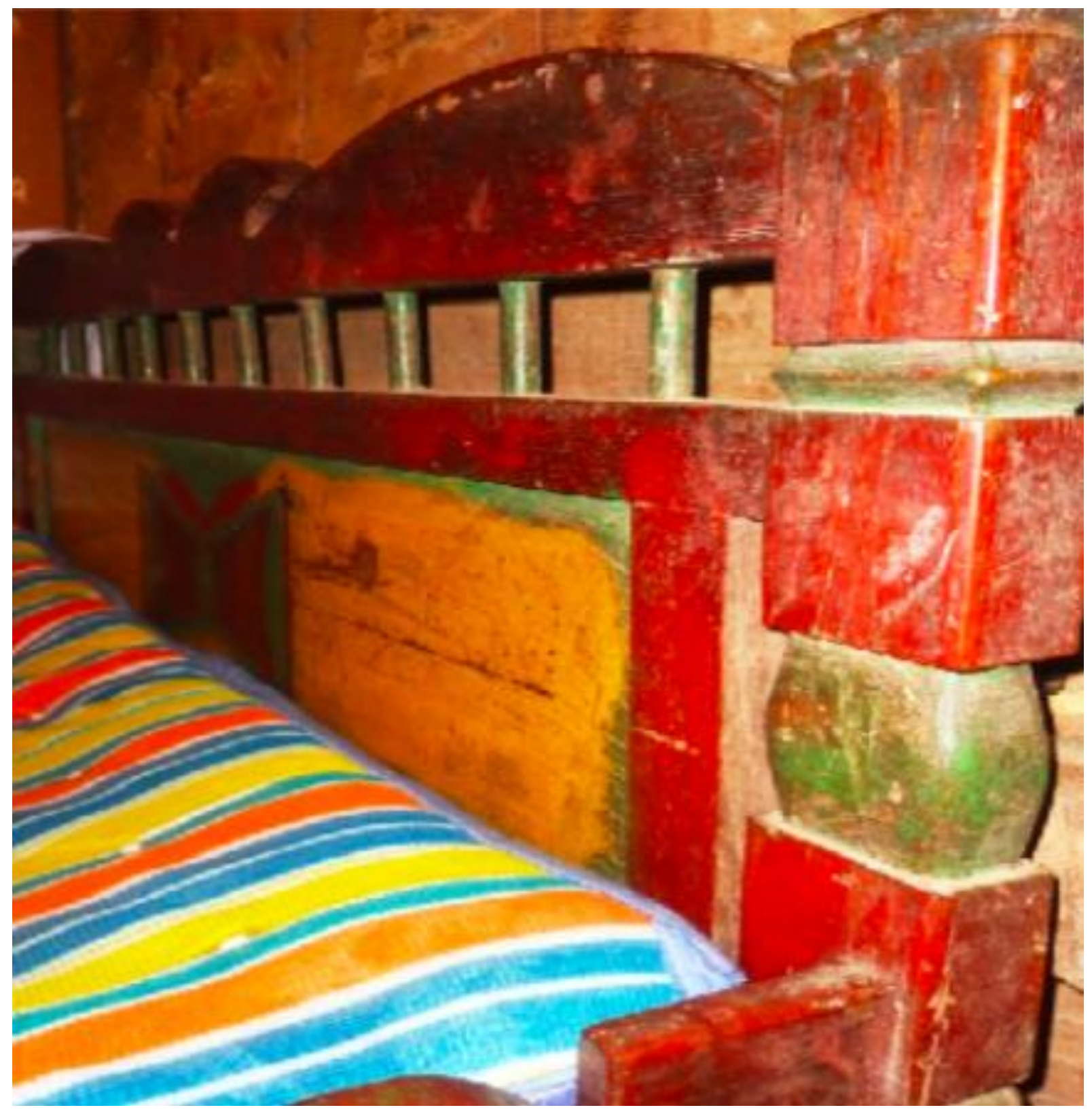

Número de la obra: 11

Título: "sobre camas"

Autor: Yenny Banesa Bonilla

Lugar: Zona rural municipio de Baraya

Finca: La Siria

Fecha de captura: Marzo 3 de 2014

Técnica: Fotografía digital

Fuente: Bonilla, Y. B. (2014). Sobre camas. Una mirada estética popular del municipio de Baraya. Tesis de pregrado. Universidad Surcolombiana. Neiva, 2014. p.80. 